\title{
Diversity of endophytic and rhizosphere bacteria from pineapple (Ananas comosus) plant in semi-arid ecosystem
}

\author{
RAHAYU FITRIANI WANGSA PUTRIE ${ }^{1,2, \bullet}$, I NYOMAN PUGEG ARYANTHA ${ }^{1}$, IRIAWATI ${ }^{1}$, \\ SARJIYA ANTONIUS ${ }^{3}$ \\ ${ }^{1}$ School of Life Sciences and Technology, Institut Teknologi Bandung. Jl. Ganesa 10, Bandung 40132, West Java, Indonesia \\ ${ }^{2}$ Research Center for Biotechnology, Indonesian Institute of Sciences. JL. Raya Bogor Km. 46 Cibinong, Bogor 16911, West Java, Indonesia. \\ Tel.: +62-21-8754587, Fax.: +62-21-8754588, ^email: rahayufwputrie@gmail.com; raha009@lipi.go.id \\ ${ }^{3}$ Research Center for Biology, Indonesian Institute of Sciences. Jl. Raya Bogor Km. 46 Cibinong, Bogor 16911, West Java, Indonesia
}

Manuscript received: 25 April 2020. Revision accepted: 15 June 2020.

\begin{abstract}
Putrie RFW, Aryantha INP, Iriawati, Antonius S. 2020. Diversity of endophytic and rhizosphere bacteria from pineapple (Ananas comosus) plant in semi-arid ecosystem. Biodiversitas 21: 3084-3093. The natural environment majorly influences plant microbiome diversity, in terms of endophytic and rhizobacteria. Pineapple (Ananas comosus L. Merr) has been identified as one of host plants with a capacity to grow in semiarid ecosystems. The intrinsic microbial symbiont composition is recognized in a specific ecological niche. The scientific information available is limited to the inoculants' ability to reduce plant stress, although the diversity in a semiarid ecosystem has not been studied. The aim of this research is to investigate the diversity of endophytic and rhizosphere bacteria obtained from pineapple in a semiarid ecosystem. A total of 117 culturable microbial isolates, dominated by Gram-positive bacteria were obtained from pineapple (88) and rhizosphere (29). The metagenomic analysis showed higher diversity and abundance in rhizospheres compared to the endophytes. Actinobacterium was identified as the most populated colony, while Proteobacterium was more prominent in the host plant. The Bacillus genera were recognized as the dominant symbiont genus. Therefore, the culturable isolates were further developed as candidate inoculants for microbe databases. This is expected to be useful in broadening theoretical knowledge.
\end{abstract}

Keywords: Diversity, endophyte, metagenomics, pineapple, rhizobacteria

\section{INTRODUCTION}

Semiarid regions are areas characterized by water scarcity and low-frequency rainfall intensity. The stability of plants present in this ecosystem is highly dependent on the use of rainfall spatial distribution and the effectiveness of other water resources (Huang et al. 2019). Also, evaporation is a dominant factor of the hydrological cycle, limited by the lesser groundwater infiltration, and influenced by low rainfall (Suarez et al. 2015). The expanse of semi-arid regions increases with different patterns, as seen on the American continent, formed from arid regions that become wetter, compared to the Eastern Hemisphere, where semi-aridity develops from consistent dry out of sub-humid/humid areas (Huang et al. 2015).

Some plants including agave, pineapple, aloe vera, and cactus grow in arid and semiarid lands, and have been adapted to grow well, despite the limited water and nutrition (Stewart 2015). These species elicit complex cellular and molecular responses needed to avert damages and survive when under stress, including the phenomenon of morphological, developmental, physiological and biochemical changes (Fahad et al. 2015). Specifically, morphological changes ensue in dry and semi-arid plants, characterized by the thin root systems and the formation of succulent body's for water storage. These plants also comprise of special metabolic pathways needed for growth, including Crassulacean acid metabolism (CAM) identified in Asparagaceae, Aloaceae, Cactaceae, and Bromeliaceae, known to prevent water loss during the day (FoncesaGarcia et al. 2016; Davis et al. 2019).

Furthermore, plant response to stress is influenced by the microbiome community structure and composition (Naylor and Coleman Derr 2018). This improves the resilience capacity, especially at the root, and is directly dependent on water pressure (Rolli et al. 2014). The microbiomes include endophytes and rhizobacteria, and are generally more dominated by bacteria than fungi. Moreover, the composition depends on the abiotic environment, host specifications, and the various microorganisms present (Liotti et al. 2018; Paredes et al. 2018).

Researches related to the microbiome of dry environments have been sparsely explored. However, this information is necessary to understand the interactions between microbial communities and plants under limiting conditions of nutrition and water (Khan et al. 2020). The studies related to microbiome diversity in arid and semiarid ecosystems have only been reported for several plants. These include Cactaceae, known to comprise a spread of species-rich succulent plant families. Symbionts of Cacti are vertically inherited to promote the plant growth and drought tolerance for the fitness of holobiont (FoncesaGarcia et al. 2016). However, there is a need to explore the plants-microbiome relationship, in terms of interactions, community contexts, and effects, in order to provide important information for the management of stressful 
agriculture (Vimal et al. 2017). A recent study reported by Khan (2020) was concerned with the rhizosphere fungi and bacteria obtained from Adenium obesum, Aloe dhufarensis, and Cleome austroarabica plants of dry environment origin, using next-generation sequencing (NGS) techniques. The findings illustrate the dependence of soil microbial community variations on the location, plant host, and soil conditions. In addition to plant genotype factors, microbial diversity also facilitates greater resistance towards stressful conditions, and specific members of the bacterial population are also possibly used to predict phenotypes in the environment (Vimal et al. 2017; Paredes et al. 2018).

Microbial symbionts, including endophytic and rhizobacteria, have been known to play an important role as a plant growth promoters by different mechanisms. This is achieved through an increase in soil fertility, participation in antioxidant activities and phenylpropanoid metabolism, plants hormone production, phytopathogen control, nitrogen fixation, phosphate solubilization, and stress activation reduction (Glick 2012; Fahad et al. 2015; Liotti et al. 2018; Ek-Ramos et al. 2019). In addition, rootassociated bacterial communities are required to maintain the hosts' health in complex relationships. The contexts of diversity and abundance are dependent on several factors, including local soil chemistry, plant genotype, phenotype, and perturbations, e.g., drought in the surrounding abiotic environment. Under these conditions, root bacterial communities play important roles, both directly, by modulating moisture availability and indirectly, by altering soil chemistry, and also plant phenotypes (Naylor and Coleman-Derr 2018).

Pineapple (Ananas comosus L. Merr) as one of several plants characterized by the capacity to grow in semiarid ecosystem (Davis et al. 2019). However, related studies on the microbial diversity have not been reported, especially in the Nusa Tenggara Province, Indonesia. The geographical location of Indonesia in the tropical climate, allows for greater plant microbiome diversity, uncommon to various locations. Furthermore, isolates from the dry areas tend to be more diverse than from samples from the normal arid environment (Khan et al. 2020). The data available about pineapple microbiome are only related to the quantification and comparison of culturable microorganism diversity existing in rhizosphere soils and in the internal network of Ananas spp. obtained from three different environments in Brazil, using BOX and ERICPCR reactions: natural forest, commercial cultivation areas, and ex-situ conservation areas Pineapple Germplasm Bank (Pineapple GB) (Souza et al. 2019). Also, the associated microbes are assumed to be unique and possess special specifications needed for improvement of plant tolerance towards drought in a semiarid ecosystem (Arif et al. 2017; Khan et al. 2020; Mohammadipanah and Wink 2016).

The 16S ribosomal RNA (rRNA) gene sequencing is widely used in comparing microbial communities among samples acquired from various natural or endozoic environments, including soil, water, host intestine, structures, functional characteristic, etc. (Lunberg et al. 2013; Akinsanya et al. 2015; Bukin et al. 2019; Deng et al.
2019). These are housekeeping genetic markers for bacteria, based on the presence in most species, characterized by an unchanged function over the course of evolution, and a size of around $1.500 \mathrm{bp}$ is appropriate for bioinformatic purposes (Janda and Abbott 2007). Moreover, the vital purpose of microbial diversity in the environment is poorly understood, as only $5 \%$ of bacterial species have been identified. The metagenomic sequencing technologies, using nextgeneration sequencing (NGS) from the $16 \mathrm{~S}$ rRNA hypervariable regions, enables rapid determination of composition and diversity in microbial communities. Additionally, NGS technology is also effectively used during the investigation of microbiomes in plant tissues (Akinsanya et al. 2015). Also, an increase in the number of reads, compared to the Sanger method confirms the appropriateness for research, alongside the advantageous metabarcoding properties available for unculturable microbes. This is required to precisely and comprehensively display data on diversity and abundance (Bukin et al. 2019).

The diversity of plant endophytes, focusing on the manner of interaction between plants and other organisms have not been intensely and carefully examined, as only about 1-2\% of all are known and studied for inherent endophytic composition (Strobel 2018). The related scientific reports on possible microbes and rhizosphere in semiarid plant species show the effect of reducing host stress, although information on diversity, especially for pineapple plants in this ecosystem has not been reported. Therefore, this study aims to investigate the diversity of endophytic bacteria and rhizosphere from pineapple obtained in a semiarid ecosystem.

\section{MATERIALS AND METHODS}

\section{Isolation of culturable endophytic and rhizosphere bacteria from pineapple plants}

The samples of pineapple plants and rhizosphere soils used were obtained from the semiarid regions in East of Nusa Tenggara, Eastern Indonesia. This dry climate is comprised of $72 \%$ rocky hills and mountains. Also, it is known to feature relatively low rainfall of less than 2.000 $\mathrm{mm}$ per year. This is known to cause limited water availability and extreme drought levels, compared to West Nusa Tenggara (Mulyani et al. 2014). The plant parts isolated for the endophytic component include the roots, stems rosettes, leaves, crowns, fruit stalks, and fruit. The microbe isolation method used was according to Tomita (2003). The plant samples were cleaned of impurities with running water, and cut into sizes $1 \times 1 \mathrm{~cm}$. Therefore, a repeat washing was performed with running water for 10 minutes, followed by drying with sterile tissue in a Petri dish. Furthermore, surface sterilization was performed using $75 \%$ ethanol for 2 minutes and then dried, before cutting again into smaller sizes with a sterile knife, followed by placement in nutrient agar (NA) media. The medium was further divided into 4 treatments, including (i) NA plus nystatin (ii) NA plus nystatin and plant extract (iii) NA (iv) NA plus plant extract. Meanwhile, the isolation of culturable rhizobacteria from soil samples was performed 
using a multilevel dilution method of up to $10^{-8}$, and each isolate obtained was then purified with the streak quadrant in NA medium. Subsequently, the respective medium was incubated at $28-30^{\circ} \mathrm{C}$ for 24 hours to optimize the growth.

\section{Gram identification by using 4\% Potassium Hydroxide (KOH) method}

Gram identification is an important and useful technique needed for the categorization of bacteria into Gram-positive or negative groups (Dash and Payyappilli 2016). The $\mathrm{KOH}$ technique is used as an alternative and also considered to be more efficient. The result correlation with strain types was $100 \%$ accurate (Powers 1995). Conversely, Gram staining output is often biased because of improper techniques, rapid decolorization of Grampositive samples, and bad decolorization of the Gramnegative bacterias (Dash and Payyappilli 2016). The $\mathrm{KOH}$ technique involves mixing a loopful of bacterial colony from the working culture with $4 \% \mathrm{KOH}$ over a glass slide, and the suspension was stirred continuously with a sterile toothpick for one minute, before gently pulling it up. The results were categorized as Gram-negative if a viscous suspension with string out was obtained after mixing, and positive in the absence of any visible strings visible (Powers 1995).

\section{Metagenomics with next-generation sequencing (NGS)}

The stages of NGS techniques include gDNA extraction, polymerase chain reaction (PCR), electrophoresis, purification of PCR product, sequencing, community composition characterization with operational taxonomic units (OTU's) and analysis. The total gDNA from pineapple plant samples were extracted using ZymoBIOMICSTM DNA Miniprep Kit Cat. No. D4300, while the rhizospheres were obtained with the CTAB method in combination with ethanol precipitation treatments. Therefore, the DNA of both were monitored using $1 \%$ agarose gels for concentration and purity, after diluting to $1 \mathrm{ng} \mu \mathrm{L}^{-1}$ with sterile water. The result amplification was attained using distinct regions (V3-V4) of 16S rRNA primer with amplicon sized around 150-400 $\mathrm{bp}$, as the sequences of individual hypervariable regions are useful for taxonomic classification (Akinsanya et al. 2015). The conserved regions of the sequence correlate with higher taxonomy levels, and the inverse was reported at less conserved areas, including the genus to species. These regional variations are widely adopted towards the characterization of microbial community diversity, with special emphasis on the use of large number of sequences obtained by the platform (Caporaso et al. 2011; Youssef et al. 2009). Also, PCR reactions were conducted with Phusion ${ }^{\circledR}$ High-Fidelity PCR Master Mix (New England Biolabs), while Novogen was used to perform the sequencing process.

\section{Bioinformatics analysis of sequencing data}

The initial steps to analyze species diversity include quality control and removal of barcode and primer sequences, followed by clean reads after chimera filtration, and quality control sequence length distribution. Therefore, all the effective reads were grouped by $97 \%$ DNA sequence similarity into Operational Taxonomic Units (OTU's), to achieve microbial species estimates, and reduce calculation complexities (Lunberg et al. 2013). Based on the results of OTU's analysis, clustering, and the research requirements, the table generated was normalized. Furthermore, the common and unique information obtained for different samples (groups) were then analyzed and the outcome was used to construct Venn diagrams. This was followed by the random selection of sequences from both samples. Therefore, the number of represented species is counted to construct a rarefaction curve, and together with the rank abundance curves, both are widely used to indicate sample biodiversity. The steep curves indicate that numerous species remain undiscovered, while the flatter ones denote the acknowledged identification of a credible number of samples, asides the scarce species. Moreover, rank abundance curve is also used to describe relative species abundance, and visualize species richness as well as evenness. In addition, data on effectiveness, frequency, and annotations read from different samples were collected during the OTU's construction, and the interactive display between species abundance and composition is described in the heat map. Subsequently, tree graphs of species annotation were constructed for each group. The GraPhlAn software was then used to generate the KRONA circle, in order to visualize the analysis result. These tools allow for the intuitive explorations of relative abundance and confidence within the complex hierarchies of a metagenomic approach (Ondov et al. 2011). The top 100 genera of the two existing microbiomes were evaluated with the evolutionary tree, while phylogenetic analysis explains the evolutionary history and the association between groups of organisms. The molecules selected tend to greatly influence the analysis result, and the presence of a distant relationship implicates the need to make a choice at lower evolutionary levels (Horiike 2016).

\section{RESULTS AND DISCUSSION}

\section{Endophytic and rhizobacteria culturable isolates}

A total of 117 culturable bacterial isolates, comprising 29 and 88, were respectively obtained from the isolation result of rhizosphere soils and pineapple plants. Basis to consider them separate isolates were different morphological colonies. In addition, the endophytic isolation was acquired from six plant parts, as shown in Figure 1. The rosette stems contain the most significant amount of endophytes. This part located with close proximity to the root is important in the transition of nutrients. This isolation yield from the same plant part and species, planted in different sites, makes it possible to evaluate differences in the numbers obtained by Bind and Nema (2019). The higher amounts were acquired in the stem of the pigeon pea at the Damoh sample compared to the root, although most other isolates were obtained from the root. In addition, Mentha arvensis plants comprise the most significant quantity of endophytes in stems (Anjum and Chandra 2015). The results indicating the principal 
influence of the site, as well as plant compartments and species on the microbial communities (Foncesa-Garcia et al. 2016).

\section{Gram-positive culturable bacteria as symbiont in pineapple plant}

Based on the results, rhizospheres and pineapple plants are dominated by Gram-positive culturable bacteria, with about $79.3 \%$ and $88.6 \%$, respectively. Figure 2 shows the total classification from each sample and the majority of culturable species were Gram-positive bacteria. Similar outcome was reported by Anjum and Chandra (2015), in a study focused on 35 endophytic culturable bacteria isolated from four plant types, including Catharanthus roseus, Ocimum sanctum, Mentha arvensis, and Stevia rebaudiana, as well as the soil. About $95 \%$ of the total bacterial isolates originated from the soil are Gram-positive (Silva and Nahaz 2002). Bacillus subtilis possess the ability to thrive in environments with extremely dry conditions. This results from the ability to produce more biofilms with $55.4 \mathrm{~nm}$ thickness, compared to $22.4 \mathrm{~nm}$ recorded in Pseudomonas aeruginosa (Mai-Prochnow et al. 2016). Furthermore, they are included as one of the most potent producers of new compounds needed for agricultural applications. The isolates obtained from extreme environmental conditions demonstrate good plant adaptation enhancement properties. These properties are due to the gene capacity to produce specific adaptation metabolites (Ek-Ramos et al. 2019).

The results obtained using the isolation method only showed a small portion of the total microbiome found in pineapple plants. This was due to the small portion of microbes grown in conventional synthetic culture, encompassing only about $<10 \%$ from nature (Sarhan et al. 2019). More complete data is obtainable from the results of metagenomic studies, which possibly reveals the basic processes related to structure, diversity, abundance, and population dynamics of the microbial communities existing in nature. This new technology greatly contributes to the development of biotechnological applications in human life, through the discovery of new genes, enzymes, pathways and bioactive molecules (Alves et al. 2018).

\section{Diversity and abundance of pineapple bacteria in semi- arid land}

The composition of plant symbiont is selected based on the root system. This is characterized by the capacity to maintain plant growth and development under limited water conditions. Furthermore, the technique is useful to support the ultimate survival of host plants under extreme circumstances (Marasco et al. 2012). The plant endophytes possess the genes required to produce specific metabolites, which play an important role in promoting growth. This is achieved through the enhancement of immune response, antioxidant activity, and phenylpropanoid metabolism. Also, a good system for plant survival results from the combination of activation pathways (Ek-Ramos et al. 2019).

The results of metagenomic analysis identified the presence of 911 bacterial OTU's in the rhizosphere. Furthermore, 191 were reported in both the soil and pineapple plants, while 53 were not found in the rhizosphere, as shown in Figure 3. This phenomenon occurs possibly because of the colonization transmission from the environment to the pineapple plants. The endophytes were obtained through the generations, both horizontally and vertically, from the environment,. The transmission route for plant colonization was achieved horizontally through soil, atmosphere, and insect, vertically through seeds and pollen. Moreover, horizontal endophyte root colonization from the soil was recognized as the beststudied route and perhaps the most important after comparing it with others (Frank et al. 2017). The transmission process is also influenced by the age of host plants or leaf, canopy cover, seasonal factors, and the presence of other microorganisms, including environmental pathogens (Strobel 2018). Furthermore, microbiota distribution from soil to other plant parts is needed to maintain growth (Souza et al. 2019).

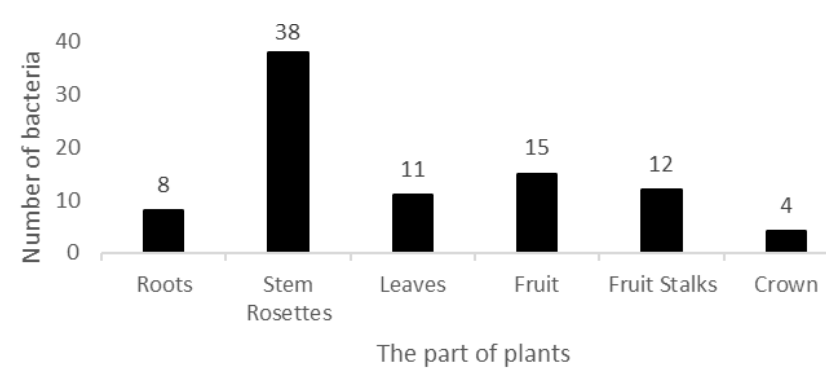

Figure 1. Abundance of culturable endophytic bacteria in pineapple plants
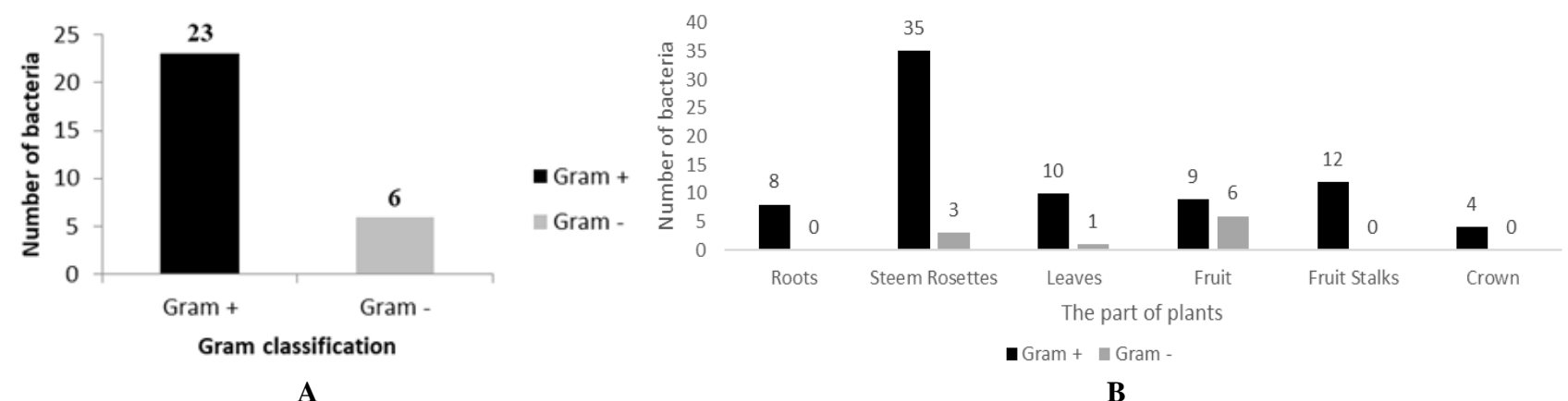

B

Figure 2. Abundance of Gram + and Gram - Bacteria from: A. Rhizosphere, B. Pineapple plants 


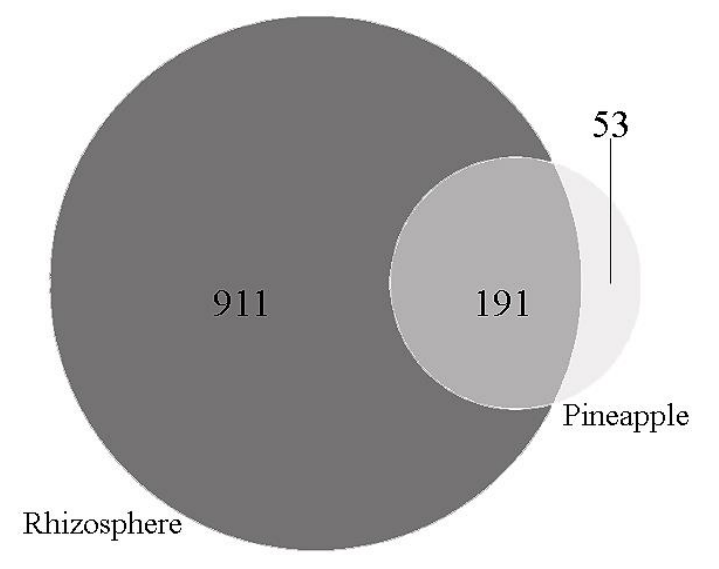

Figure 3. Venn diagram of the diversity of bacterial based on OTU's

Diversity based on OTU's and bacterial species abundance in both samples was denoted in both rarefactions and rank abundance curves (Figure 4). The outcome was higher in the rhizosphere, compared to the pineapple plants. This was because soil in semiarid ecosystems tends to contain more distinct microbes than those present in endosphere tissue (Foncesa-Garcia et al. 2016). Furthermore, there was a marked decline in the abundance gradient from the soil to the plants tip, hence elevated part possessed lesser microbes (Souza et al. 2019). This soil microbiome diversity is higher because of the important role played as the main controller in the ecosystem, related to activities based on organic matter mineralization rate. They have also been applied in determining of biological parameters related to soil quality (Mhete et al. 2019).

The top 10 species in the different taxonomic ranks were selected to form the distribution histogram of relative abundance, as shown in Figure 5. The major bacterial population present in the pineapple plants includes
Proteobacteria and Cyanobacteria. Huang (2018) reported a similar outcome, stipulating both phyla as dominant in plants. However, Cyanobacteria are more abundant in leaves than roots, in contrast with Proteobacteria. These plants require exposure to the same group of microbes, to maintain certain tissue functions (Souza et al. 2019). Despite the limited nutrient source, compared to rhizosphere areas, the inherent compartments are relatively more stable (Maranto-Barrios et al. 2018). This allows for the dominance of Cyanobacteria in leaves. This symbiont has the capacity for nitrogen fixation, production of amino acids, polysaccharides, and phytohormones, expected to act as elicitor molecule required to influence plant gene expressions, therefore changing the plant phytochemical composition (Singh 2014). Conversely, the roots are dominated by Proteobacteria group, particularly from the Gammaproteobacteria and Flavobacteriia classes, although Alphaproteobacteria and Cytophagia have also been identified in lesser amounts (Furtado et al. 2019).

Furthermore, rhizosphere bacterial abundance is known to be more diverse compared to the pineapple plant. The results show the dominance of Actinobacteria in the population, which was similar to the study outcome of Souza et al. (2019). The Actinobacteria genus has the ability to withstand extreme environments, and are known also commonly identified in large numbers under arid conditions. This phenomenon is due to the adaptability towards relatively high temperatures, salt concentrations, and radiation. The polyketide synthase (PKS) and nonribosomal peptide synthetase (NRPS) genes were detected in the xerophile and aerotolerant actinobacteria genes. Meanwhile, several bioactive compounds, including ectoin and hydroxyectoin produced by microorganisms are potentially used in industries and for agriculture purposes (Mohammadipanah and Wink 2016). In addition, the endophytic bacteria diversity, metabolite production ability, and adaptability to stress generate the assumption as a potential source of new metabolites (Ek-Ramos et al. 2019).

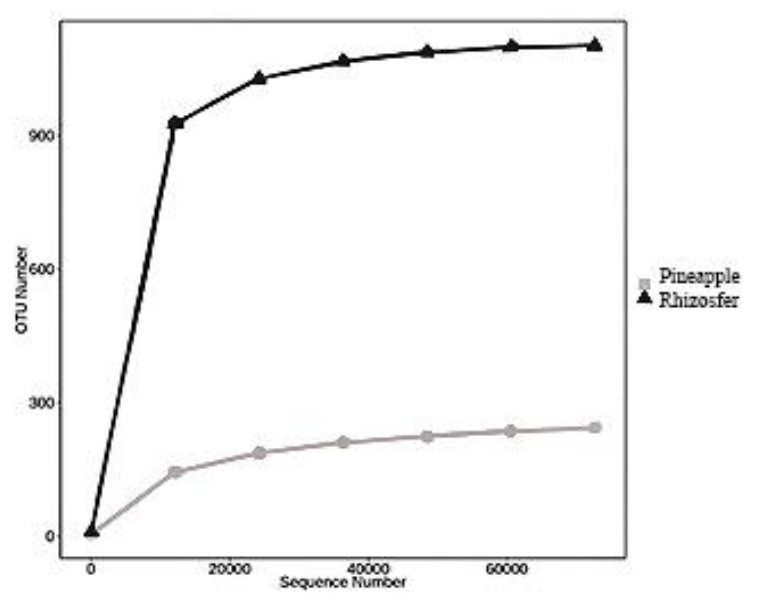

$\mathbf{A}$

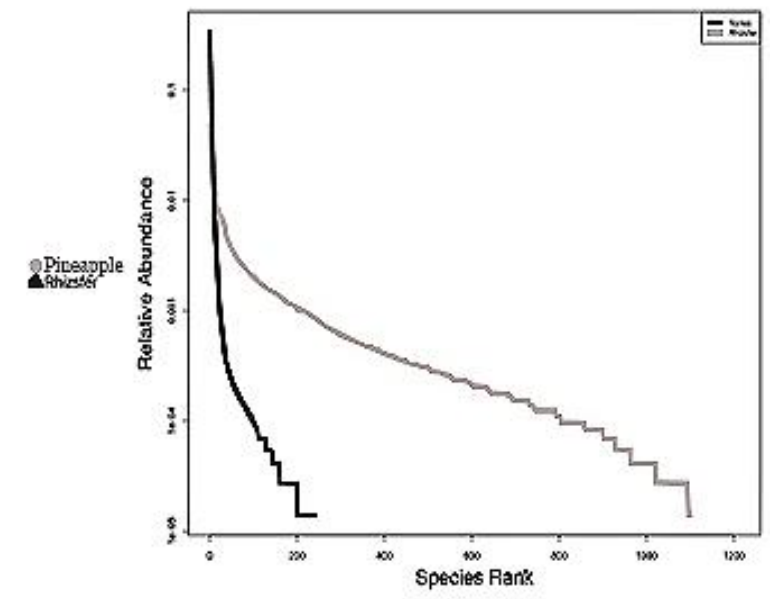

B

Figure 4. Biodiversity of the rhizosphere and pineapple plant based on: A. Rarefraction curve, B. Rank abundance curve 


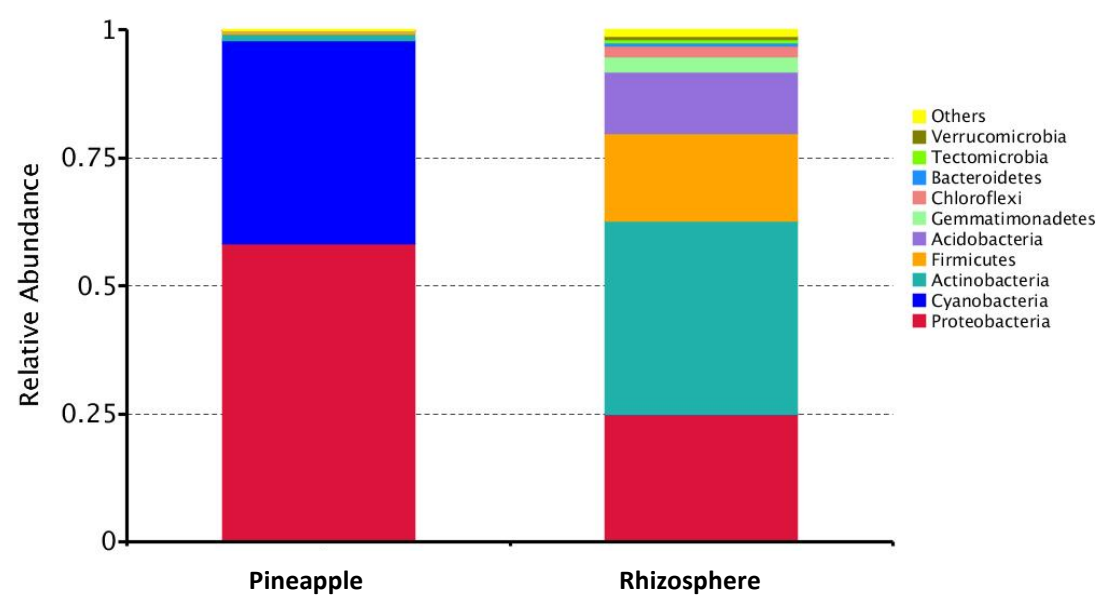

Sample Name

Figure 5. Relative abundance of the top 10 species in the different taxonomic ranks

The soil bacterial community characteristics, as well as the diversity, compositions, and functions also relate to carbon (C) and nitrogen (N) cycling. Therefore, samples with higher $\mathrm{C} / \mathrm{N}$ ratio contain a more significant bacteria population (Deng et al. 2019). The value measured in this study is 10 , as semi-arid soils are always relatively low. According to Swangjang (2015), ratio of less than 20 is classified as nutrient-poor due to the faster release of mineral $\mathrm{N}$ during the decomposition process. In addition, $\mathrm{C} / \mathrm{N}$ is a key regulation parameter of mineralization, commonly used to accurately determine the decomposition rate of organic matter. This event results in the release or immobilization of nitrogen, particularly where optimum mineralization occurs at a $\mathrm{C} / \mathrm{N}$ ratio of 25 .

Another influencing factor of soil microbiota abundance and diversity is root exudate. This is estimated to improve nutrient richness at the root area, due to the numerous chemical components. In addition, they also serve as chemotactic signals needed to attract bacteria. The phenomenon triggers rhizosphere microbial colonization, resulting in plant microbiome modification. (Souza et al 2019). Also, genotype factors, plant organs, and geographical environmental conditions influence the structure, composition, and function of endophytic bacterial communities (Liotti et al. 2018). Meanwhile, the natural integrity of the ecosystem influences microbial diversity. Hence, habitats disturbed by land use management demonstrate altered soil characteristics (Mhete et al. 2019). This is observed in terms of soil bacteria abundance and assortment in the stressed environment, which decreases by about $10^{4}$ from the normal $10^{8}-10^{9}$ cells per gram of soil. (Glick 2012).

Figure 6 shows Actinobacteria to be the dominant group in the rhizosphere soil, characterized by Thermophilla as the major genus. Kurapova et al (2012), recorded a similar outcome, based on the study conducted on desert lands from Mongolia. However, the thermotolerant Actinomycete and thermophilic Actinomycetes were more dominant than the mesophilic
Actinobacteria. The Streptomyces, Micromonospora, Actinomadura, and Streptosporangium are members of the order Actinomycetales, comprising most species in desert land. However, Actinobacteria, Proteobacteria, Firmicutes, and Acidobacteria are also abundant in the rhizosphere of pineapple plants. The metagenomic research conducted by Mhete showed the domination of Proteobacteria, Actinobacteria, Firmicutes, and Acidobacteria phylum in soil, while Actinobacteria and Proteobacteria are more common in plants and rhizospheres. Specifically, Proteobacteria are involved in the carbon, nitrogen, and sulfur cycles of various soil types, including rhizospheres, saline, and semi-arid regions (Khan et al. 2020). The Firmicutes phylum has also been affiliated with the production of typical metabolites needed for biocontrol and plant growth (Mhete et al. 2019). Also, Acidobacteria plays a role in this process due to the number of ecological capabilities, including the use of nitrite as a source of $\mathrm{N}$, good adaptability to changes in macroelements and nutrients, soil acidity, expression of active transporters, and exopolysaccharide (EPS) production (Ek-Ramos et al. 2019). Based on metagenomic study, this phylum is characterized by 9 out of 20 open reading frame (ORF) qlcA genes, assumed to play a role in lactonase activity as well as polyketide synthesis during metabolite production (Kielak et al. 2016).

The top 100 genera from the two existing microbiomes were selected, and the evolutionary tree was drawn using an aligned representative sequence, and the relative abundance of each genus was shown in Figure 7. In addition, the genus Bacillus was estimated to be the highest in the rhizosphere, due to the characteristic resistance towards drought stress. Research on this phenomenon has been performed on Platycladus orientalis seedlings. The cytokine hormones played a role in reducing stress and suppressing plant shoot growth. Based on this current research, Bacillus is also able to survive under dry soil conditions with low $\mathrm{C} / \mathrm{N}$ ratio, alongside the low water content of $17.43 \%$ estimated from physicochemical 
evaluations (Liu et al. 2013). Specifically, Bacillus cereus GS6 is reported capable of surviving in nutrient-poor soils present in arid and semiarid areas, and further increases symbiosis efficiency in growing soybean plants. Also, there is a great potential for phosphate dissolution and mobilization, following the release of carboxylates in insoluble phosphate. is also reported to be able to survive in nutrient-poor soils in arid and semiarid areas (Arif et al. 2017).

There is no significant difference in the abundance of each genus present in most pineapple plants. Also, numerous unidentified chloroplasts were reported in the NGS analysis result. This phenomenon was probably due to the high affinity of many primary marker pairs in bacteria for non-target DNA from plastid (chloroplasts) and plant mitochondrial, due to the homologous features (Beckers et al. 2016). Furthermore, Methylobacterium and
Pseudomonas were identified as the most abundant genus of the Proteobacteria phylum. These are commonly reported as endophytes in plants, characterized by the capacity to produce alkaloids, flavonoids, and steroids. The M. radiotolerans MAMP 4754 species isolated from the seeds of the medicinal plant Combretum erythrophyllum are able to produce secondary metabolites with antimicrobial and antioxidant activity (Photolo et al. 2020). In addition, Pseudomonas aeruginosa PAO1 and Pseudomonas geniculata ATCC 19374 strains also produce hydrogen cyanide (HCN), indole acetic acid (IAA), siderophore and phosphate solubilizing, while the inoculation to corn plants show the possibility of improved productivity, alongside increased carbohydrate and oil content in corn kernels obtained under desert condition (Omer 2016).

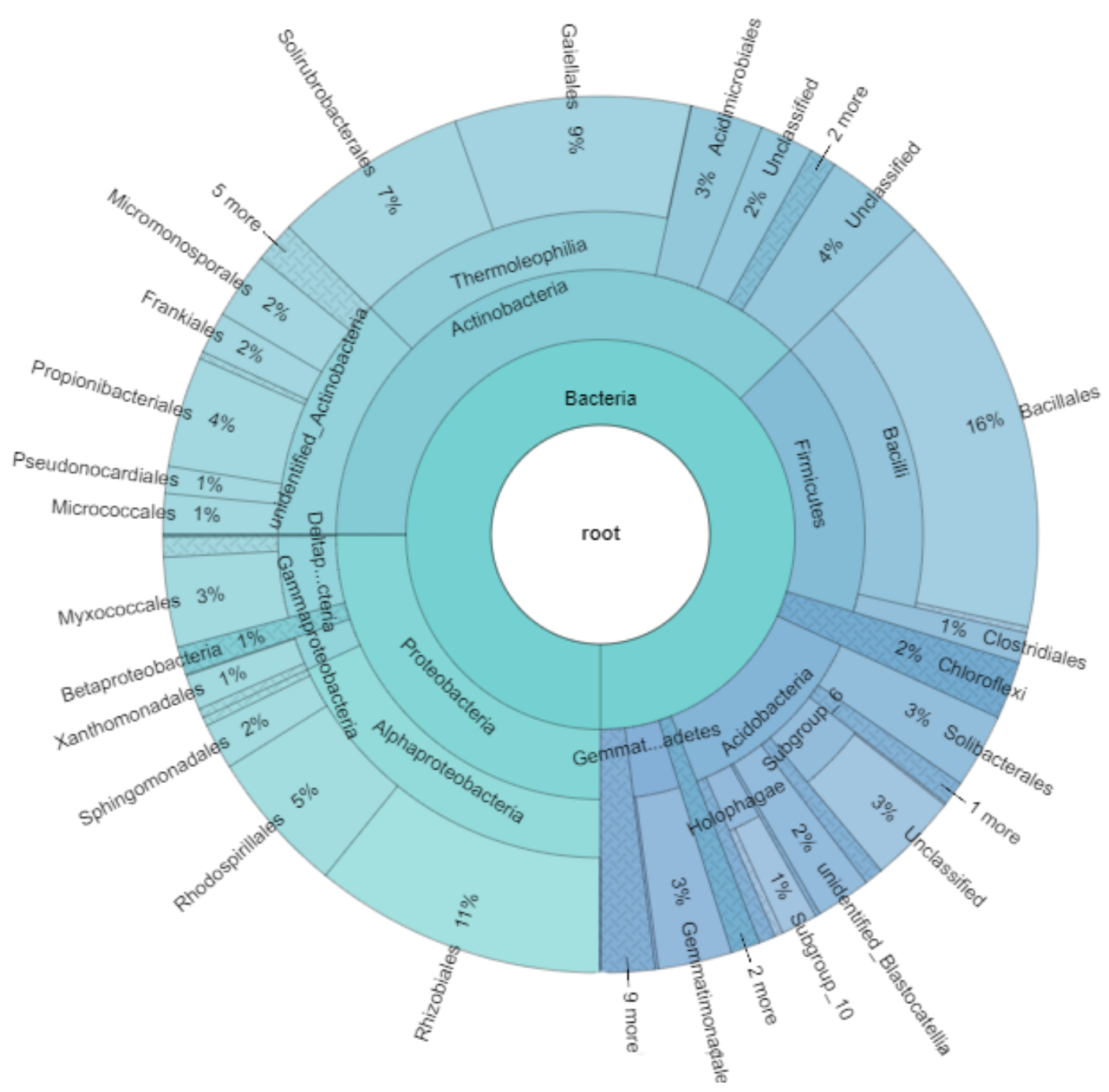

Figure 6. Abundance of bacteria in rhizosphere of pineapple 


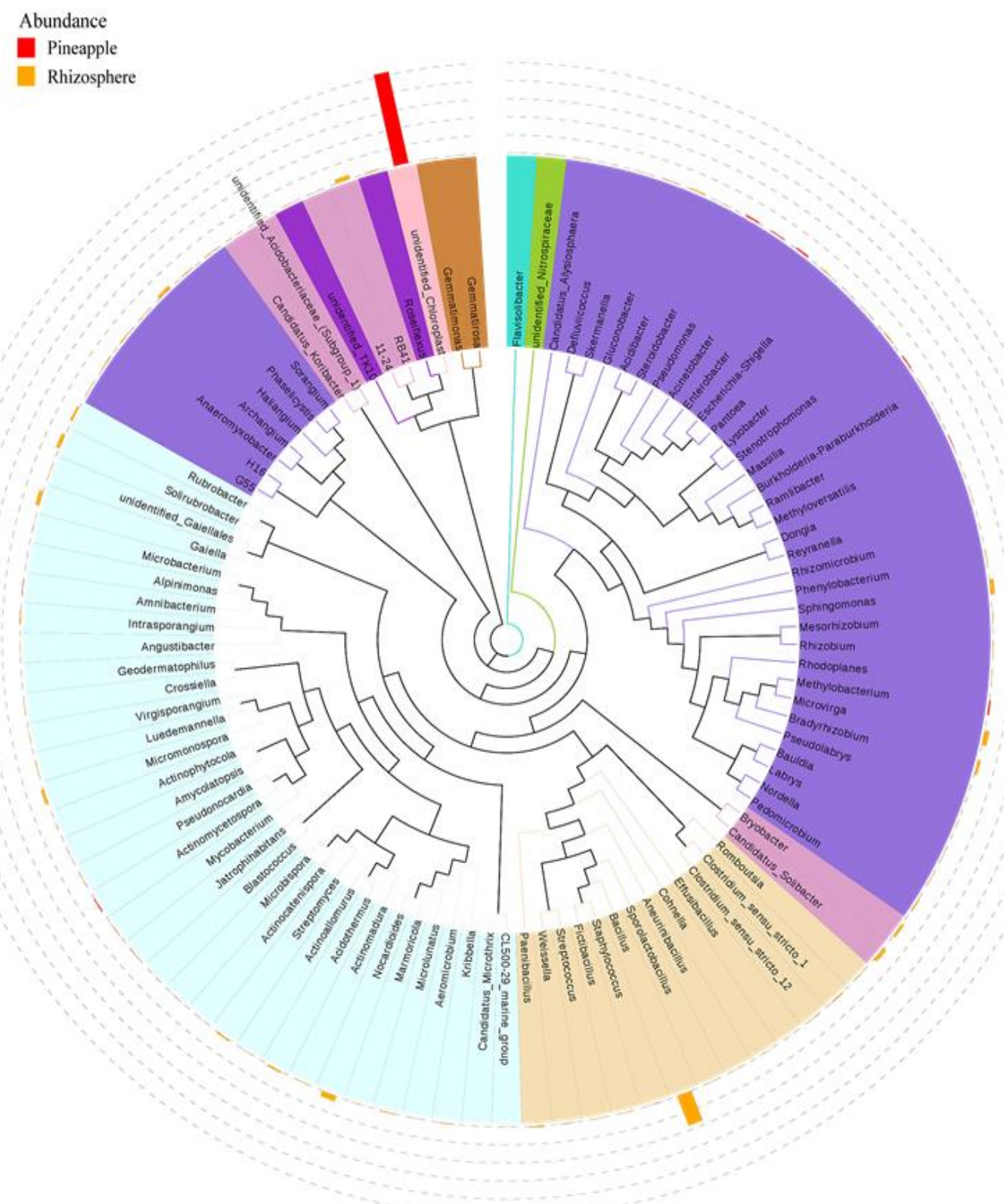

Figure 7. The relative abundance of each genus from plant and rhizosphere microbiome. Different branches color represents distinct phyla, and the respective abundance was displayed outside the circle, with dissimilar colors signifying different groups

In conclusion, the diversity of microbiome from semiarid plants, including pineapple is influenced by the plant compartments and species, as well as the environment. The selected symbionts species composition help maintain host plant survival during stressful abiotic conditions, including drought. Based on the result, there is a potential to develop a collection of endophytic and rhizobacterial isolates as biological agents to improve plant growth, after gaining profound understanding of the respective abilities. Moreover, microbiome diversity is also applicable in the development of wider knowledge for theoretical database.

\section{ACKNOWLEDGEMENTS}

The authors are grateful to the Ministry of Research, Technology and Higher Education, Republic of Indonesia, for funding this research, as well as all staff and members of Microbiology Laboratory of the Research Center for Bioscience and Biotechnology, and the Instrumentation Laboratory of School of Life Sciences and Technology of Bandung Institute of Technology, Bandung, Indonesia for the facilities and support provided. 


\section{REFERENCES}

Akinsanya MA, Goh JK, Lim SP, Ting ASY. 2015. Metagenomics study of endophytic bacteria in Aloe vera using next-generation technology. Genom. Data 6: 159-163.

Alves LF, Westmann CA, Lovate GL, Viana de Siqueira GM, Borelli TC, Guazzaroni ME. 2018. Metagenomic approaches for understanding new concepts in microbial science. Int J Genomics 2018: 2312987. DOI: $10.1155 / 2018 / 2312987$.

Anjum N, Chandra R. 2015. Endophytic bacteria: optimization of isolation procedure from various medicinal plants and their preliminary characterization. Asian J Pharm Clin Res. 8: 233-238.

Arif MS, Riaz M, Shahzad SM, Yasmeen T. 2017. Phosphorus-mobilizing rhizobacterial strain Bacillus cereus GS6 improves symbiotic efficiency of soybean on an aridisol amended with phosphorusenriched compost. Pedosphere 27: 1049-1061.

Beckers B, Op De Beeck M, Thijs S, Truyens S, Weyens N, Boerjan W, Vangronsveld J. 2016. Performance of 16S rDNA primer pairs in the study of rhizosphere and endosphere bacterial microbiomes in metabarcoding studies. Front Microbiol 7: 650. DOI: 10.3389/fmicb.2016.00650

Bind M, Nema S. 2019. Isolation and molecular characterization of endophytic bacteria from pigeon pea along with antimicrobia evaluation against Fusarium udum. Appl Microbiol 5: 1000163. DOI: 10.35248/2471-9315.1000163.

Bukin YS, Galachyants YP, Morozov IV, Bukin SV, Zakharenco AS, Zemskaya TI. 2019. The effect of $16 \mathrm{~S}$ rRNA region choice on bacterial community metabarcoding results. Sci Data 6: 190007. DOI: 10.1038/sdata.2019.7.

Caporaso JG, Lauber CL, Walters WA, Bergs-Lion D, Lozupone CA, Turnbaugh PJ, Fierer N, Knight R. 2011. Global patterns of $16 \mathrm{~S}$ rRNA diversity at a depth of millions of sequences per sample. Proc Natl Acad Sci USA 108: 4516-4522.

Dash C, Payyappilli RJ. 2016. KOH string and vancomycin susceptibility test as an alternative method to Gram staining. J Intl Med Dentistry 3: $88-90$.

Davis SC, Simpson J, Gil-Vega KC, Niechayev NA, Tongerlo V, Castano NH, Dever LV, Búrquez A. 2019. Undervalued potential of crassulacean acid metabolism for current and future agricultural production. J Exp Bot 70: 6521-6537.

Deng J, Zhang Y, Yin Y, Zhu X, Zhu W, Zhou Y. 2019. Comparison of soil bacterial community and functional characteristics following afforestation in the semi-arid areas. PeerJ 7: e7141. DOI: 10.7717/peerj.7141.

Ek-Ramos MJ, Gomez-Flores R, Orozco-Flores AA, Rodriguez-Padilla C, Gonzalez-Ochoa G, Tamez-Guerra P. 2019. Bioactive products from plant-endophytic Gram-Positive bacteria. Front Microbiol 10: 463. DOI: $10.3389 /$ fmicb.2019.00463.

Fahad S, Hussain S, Bano A, Saud S, Hassan S, Shan D, Khan FA, Khan F, Chen Y, Wu C, Tabassum MA, Chun MA, Afzal M, Jan A, Jan M T, Huang J. 2015. Potential role of phytohormones and plant growthpromoting rhizobacteria in abiotic stresses: consequences for changing environment. Environ Sci Pollut Res 22: 4907-4921. DOI: 10.1007/s11356-014-3754-2.

Fonseca-García C, Coleman-Derr D, Garrido E, Visel A, Tringe SG, Partida-Martínez LP. 2016. The cacti microbiome: interplay between habitat-filtering and host-specificity. Front Microbiol 7: 150. DOI: 10.3389/fmicb.2016.00150.

Frank AC, Guzman JPS, Shay JE. 2017. Transmission of bacterial endophytes Microorganisms 5 (4): 70. DOI: 10.3390/microorganisms5040070.

Furtado BU, Golebiewski M, Skorupa M, Hulisz P, Hrynkiewicz K. 2019. Bacterial and fungal endophytic microbiomes of Salicornia europaea. Appl Environ Microbiol 85: e00305-19. DOI: 10.1128/AEM.0030519.

Gagne-Bourque F, Bertrand A, Claessens A, Allferis KA, Jabaji S. 2016. Alleviation of drought stress and metabolic changes in timothy (Phleum pratense L.) colonized with Bacillus subtilis B26. Front. Plant Sci 7: 584. DOI: 10.3389/fpls.2016.00584.

Glick BR. 2012. Plant growth-promoting bacteria: mechanisms and applications. Scientifica 2012: 963401. DOI: 10.6064/2012/963401.

Horiike T. 2016. An introduction to molecular phylogenetic analysis. Rev Agric Sci 4: 36-45.

Huang J, Ji M, Xie Y, Wang S, He Y, Ran J. 2015. Global semi-arid climate change over last 60 years. Pharmacol Res 40(3), 211-225.
Huang J, Tagawa K, Wang B, Wen J, Wang J. 2019. Seasonal surface runoff characteristics in the semiarid region of Western Heilongjiang Province in Northeast China case of the Alun River Basin. Water 11: 557. DOI: $10.3390 /$ w11030557.

Huang Y. 2018. Comparison of rhizosphere and endophytic microbial communities of Chinese leek through high-throughput 16S rRNA gene Illumina sequencing. J Integr Agric 17: 359-367.

Janda JM, Abbott SL. 2007. 16S rRNA gene sequencing for bacterial identification in the diagnostic laboratory: pluses, perils, and pitfalls. $\mathrm{J}$ Clin Microbiol 45: 9. DOI: 10.1128/JCM.01228-07.

Khan AL, Asaf F, Abed RMM, Chai YN, Al-Rawahi AN, Mohanta TK, Al-Rawahi A, Schachtman DP, Al-Harrasi A. 2020. Rhizosphere microbiome of arid land medicinal plants and extracellular enzymes contribute to their abundance. Microorganism 8: 213. DOI: 10.3390/microorganisms8020213.

Kielak AM, Barreto CC, Kowalchuk GA, Veen JA, Kuramae EK. 2016. The ecology of Acidobacteria: moving beyond gene and genomes. Front Microbiol 7: 744. DOI: 10.3389/fmicb.2016.00744.

Kurapova AI, Zenovaa GM, Studnitsyna II, Kizilovab AK, Manucharova NA, Norovsurenc Zh, Zvyagintseva DG. 2012. Thermotolerant and thermophilic Actinomycetes from soils of Mongolia desert steppe zone. Microbiology 8: 98-108.

Liotti RG, Figueiredo MI, Ferreira da Silva G, Furtado de Mendonça EA, Soares MA. 2018. Diversity of cultivable bacterial endophytes in Paullinia cupana and their potential for plant growth promotion and phytopathogen control. Microbiol Res 207: 8-18.

Liu F, Xing S, Ma H, Du Z, Ma B. 2013. Cytokinin-producing, plant growth-promoting rhizobacteria that confer resistance to drought stress in Platycladus orientalis container seedlings. Appl Microbiol Biotechnol 97: 9155-9164.

Lundberg DS, Yourstone S, Mieczkowski P, Jones CD, Dang JL. 2013. Practical innovations for high-throughput amplicon sequencing. Nat Methods 10: 999-1002.

Marasco R, Rolli E, Ettoumi B, Vigani G, Mapelli F, Borin S, AbouHadid AF, El-Behairy UA, Sorlini C, Cherif A, Zocchi G, Daffonchio D. 2012. A drought resistance-promoting microbiome is selected by root system under desert farming. PLoS One 7(10):e48479. DOI: 10.1371/journal.pone.0048479.

Mai-Prochnow A, Clauson M, Hong J, Murphy AB. 2016. Gram-positive and Gram-negative bacteria differ in their sensitivity to cold plasma. Sci Rep 6: 38610. DOI: 10.1038/srep38610.

Mohammadipanah F, Wink J. 2016. Actinobacteria from arid and desert habitats: diversity and biological activity. Front Microbiol 6: 1541. DOI: $10.3389 /$ fmicb.2015.01541.

Mulyani A, Nursyamsi D, Las I. 2014. Acceleration of dry climate dryland agriculture development in Nusa Tenggara. Agric Innov Develop 7: 187-198.

Mhete M, Eze PN, Rahube TO, Akinyemi FO. 2019. Soil properties influence bacterial abundance and diversity under different land-use regimes in semi-arid environments. Sci Afr 7: e00246. DOI: 10.1016/j.sciaf.2019.e00246.

Naylor D, Coleman-Derr, D. 2018. Drought stress and root-associated bacterial communities. Front Plant Sci 8: 2223. DOI: 10.3389/fpls.2017.02223, [24 May 2019].

Omer AM. 2016. Role of endophytic Pseudomonas as plant growth promoters under desert conditions. Egyptian J. Desert Res. 66(2): 305-326.

Ondov BD, Bergman NH, Phillippy AM. 2011. Interactive metagenomic visualization in a Web browser. BMC Bioinformatics 12:385. 12:385. DOI: $10.1186 / 1471-2105-12-385$.

Paredes SH, Gao T, Law TF, Finkel OM, Mucyn T, Teixeira PJPL, Gonzalez IS, Fletcher ME, Powers MJ, Shank WA, Jones CD, Jojic V, Dang JL, Castrillo G. 2018. Design of synthetic bacterial communities for predictable plant phenotypes. PLoS Biol 16 (2): e2003962. DOI: 10.1371/journal.pbio.2003962,

Photolo MM, Mavumengwana V, Sitole L, Tlou MG. 2020. Antimicrobial and antioxidant properties of a bacterial endophyte, Methylobacterium radiotolerans MAMP 4754, isolated from Combretum erythrophyllum seeds. Intl J Microbiol. DOI: 10.1155/2020/9483670,

Powers EM. 1995. Efficacy of the Ryu nonstaining KOH technique for rapidly determining Gram reactions of food-borne and waterborne bacteria and yeasts. Appl Environ Microbiol 61: 3756-3758.

Rolli E, Marasco R, Vigani G, Ettoumi B, Mapelli F, Deangelis ML, Gandolfi C, Casati E, Previtali F, Gerbino R, Cei FP, Borin S, Sorlini C, Zocchi G, Daffonchio D. 2014. Improved plant resistance to drought is promoted by the root-associated microbiome as a water 
stress-dependent trait. Environ Microbiol. DOI: 10.1111/1462 2920.12439.

Sarhan MS, Hamza MA, Youssef HH, Patz S, Becker M, ElSawey H, Nemr R, Daanaa HAS, Mourad EF, Morsi AT, Abdelfadeel MR Abbas MT, Fayez M, Ruppel S, Hegazi NA. 2019. Culturomics of the plant prokaryotic microbiome and the dawn of plant-based culture media-A review. J Adv Res 19: 15-27.

Silva P, Nahaz E. 2002. Bacterial diversity in soil in response to different plants, phosphate fertilizers and liming. Braz J Microbiol 33: $304-$ 310.

Singh S. A review on possible elicitor molecules of Cyanobacteria: their role in improving plant growth and providing tolerance against biotic or abiotic stress. J Appl Microbiol 117: 1221-1244.

Stewart JR. 2015. Agave as a model CAM crop system for a warming and drying world. Front. Plant Sci. 6: 684. DOI: 10.3389/fpls.2015.00684

Strobel G. 2018. The emergence of endophytic microbes and their biological promise. J Fungi 4: 1-19. DOI: 10.3390/jof4020057.

Souza CR, Barbosa AC, Ferreira CF, Souza FVD, Rochab L, Hilo de Souza E, Santos de Oliveira SA. 2019. Diversity of microorganisms associated to Ananas spp. from natural environment, cultivated and ex situ conservation areas. Sci Hortic 243: 544-551.

Suarez VVC, Okello AMLS, Wenninger JW, Uhlenbrook S. 2015. Understanding runoff processes in a semi-arid environment through isotope and hydrochemical hydrograph separations. Hydrol Earth Syst Sci 19: 4183-4199.

Swangjang K. 2015. Soil carbon and nitrogen ratio in different land use. IPCBEE 87: 36-40. DOI: 10.7763/IPCBEE.2015.V87.7.

Tomita F. 2003. Endophytes in Southeast Asia and Japan: their taxonomic diversity and potential applications. Fungal Divers 14: 187-204.

Vimal SR, Singh JS, Arora NK, Singh S. 2017. Soil-plant-microbe interactions in stressed agriculture management: a review. Pedosphere 27: 177-192.

Youssef N, Sheik CS, Krumholz LR, Najar FZ, Roe BA, Elshahed MS. 2009. Comparison of species richness estimates obtained using nearly complete fragments and simulated pyrosequencing-generated fragments in 16S rRNA gene-based environmental surveys. Appl Environ Microbiol 75: 5227-5236. 